\title{
Anatomic Dissection of the Femoral Vein at the Bamako Anatomy Laboratory
}

\author{
Babou Ba ${ }^{1 *}$, Abdoulaye Kanté ${ }^{1,2}$, Tata Touré1 (), Moumouna Koné1, Fousseyni Guissé ${ }^{3}$, \\ Drissa Traoré ${ }^{2}$, Tièman Coulibaly ${ }^{3}$, Nouhoun Ongoïba1,2, A. K. Koumare ${ }^{1}$
}

${ }^{1}$ Laboratoire d'Anatomie, Faculté de Médecine et d'Odontostomatologie de Bamako, Bamako, Mali

${ }^{2}$ Service de Chirurgie B, CHU du Point G, Bamako, Mali

${ }^{3}$ Service de Traumatologie, CHU Gabriel Touré, Bamako, Mali

Email: *babouba20022000@yahoo.fr

How to cite this paper: Ba, B., Kanté, A., Touré, T., Koné, M., Guissé, F., Traoré, D., Coulibaly, T., Ongoïba, N. and Koumare, A.K. (2019) Anatomic Dissection of the Femoral Vein at the Bamako Anatomy Laboratory. Forensic Medicine and Anatomy Research, 7, 76-84.

https://doi.org/10.4236/fmar.2019.74012

Received: July 25, 2019

Accepted: August 30, 2019

Published: October 15, 2019

Copyright $\odot 2019$ by author(s) and Scientific Research Publishing Inc. This work is licensed under the Creative Commons Attribution International License (CC BY 4.0).

http://creativecommons.org/licenses/by/4.0/

\begin{abstract}
Objectives: The purpose of this work was to measure the dimensions of the femoral veins, to describe the affluent and the variations of the femoral veins.

Methodology: Twenty-four femoral veins of 12 fresh adult cadavers were dissected and photographed. Results: The diameter of the superficial, deep and common femoral veins was respectively $8.75 \mathrm{~mm} ; 7.60 \mathrm{~mm}$ and 13.95 $\mathrm{mm}$. The common femoral vein was $80.70 \mathrm{~mm}$ long. At the level of the superficial vein, the modal disposition was noted in $79.17 \%$; as anatomical variations, it was split in 2 cases, the presence of a collateral canal in 1 case and in 2, and it received a quadricipital muscle vein. At the level of the deep femoral vein, the modal disposition was noted in $16.67 \%$, and the anatomical variations were noted in $83.33 \%$ where it received no affluent in the femoral trine. The modal disposition was noted in $91.67 \%$ at the level of the mode of birth of the common femoral vein, in $16.67 \%$ at the level of its tributaries. The anatomical variations were noted in $8.33 \%$ in the mode of birth of the femoral vein, in $83.33 \%$ in which the common femoral vein received, in addition to the large saphenous vein, other tributaries, the most frequent of which were the quadricipital veins and the circumflex femoral veins. Conclusion: The diameter of the femoral veins is important. The main tributaries of the common femoral vein are GVS, VQ and circumflex femoral veins. The femoral variations are numerous and important to know to avoid a possible misdiagnosis in case of their thrombosis.
\end{abstract}

\section{Keywords}

Femoral Vein, Common Femoral Vein, Deep Femoral Vein, Superficial Femoral Vein, Circumflex Lateral Vein of the Thigh, Circumflex Vein Medial Thigh, Quadriceps Vein, Large Saphenous Vein, Anatomic Variation, Deep Femoral Artery 


\section{Introduction}

The femoral veins are 3 in number: the superficial, deep and common femoral veins [1]. The first two will unite to form the last one that ends below the inguinal ligament, continuing through the external iliac vein. They drain almost all the veins of the pelvic limb. Many authors [2] [3] [4] [5] do not distinguish the common femoral vein from the superficial femoral vein. According to these authors, the femoral vein has a main trunk that follows the popliteal vein and ends below the inguinal ligament, continuing through the external iliac vein, and in this case the deep femoral vein is considered a tributary of the femoral vein. In this study, the femoral veins were described as follows: the superficial vein unites with the deep femoral vein to form the common femoral vein.

The non-realization of an anatomic dissection study on the femoral veins; the frequency of their lesion in the trauma of the groin; the frequency of the localization of thrombosis at their level, the realization of the catheterization of the femoral vein in the femoral trigone for various examinations, in particular, a blood sample and the recording of the blood pressure in the cavities of the right heart and/or in the pulmonary artery, or angiography of the right heart, led us to undertake their study with the general objective of to study them by anatomic dissection. The specific objectives were to measure their dimensions, look for their tributaries and describe their anatomical variations.

\section{Methodology}

This was a prospective study carried out in the Anatomy Laboratory of the Faculty of Medicine and Ondoto-Stomatology (FMOS) of Bamako from July 26, 2018 to June 11, 2019. We dissected the femoral veins at Femoral trine level on both sides of 12 cadavers of adults, making a total of 24 femoral veins including 18 in men and 6 in women. The inclusion criteria were: fresh corpses of adults with no operative scar or traces of trauma in the inguinal regions. The exclusion criteria were: corpses with scars or traces of trauma in the inguinal regions. The approach was inguinal. For this, a cutaneous incision was made from the anterior superior iliac spine to the superior medial edge of the thigh passing through the pubic spine. A second incision started from the middle of the first until the junction of the upper $1 / 3$ and $2 / 3$ inferior of the anterior aspect of the thigh. A transverse incision was made from the lower end of the second incision. Thus, three skin flaps were dissected and folded inside, outside and above. The subcutaneous tissue was dissected for the large saphenous vein (LSV) that was dissected until it entered the common femoral vein. The screened fascia was detached, the vascular sheath was opened, the femoral vein was separated from the femoral artery. The femoral arteries and veins were dissected to the top of the femoral trine. The tributaries of the femoral veins have been dissected to the point of failure between the muscles. After these dissections, the measurements were made using a tape measure. The femoral veins were photographed before and after resection. The data was entered and analyzed on the Epi info software. 
The writing of the final document was done on Word 2016.

\section{Results}

\section{Socio-demographic characteristics of cadaveric subjects:}

The distribution of anatomical subjects by sex is summarized in Table 1 . The distribution of anatomical subjects by size is summarized in Table 2.

\subsection{Superficial Femoral Vein (SFV)}

The diameter of the superficial femoral vein was $8.75 \mathrm{~mm}$ (range: 6 and 11). Of the 24 dissections, the classical modal arrangement (the superficial femoral vein did not receive any affluent) was noted in 19 cases, i.e. $79.17 \%$. The anatomical variations were noted in 5 cases, namely 20.83\%: the femoral vein was split in 2 cases and the 2 trunks joined together forming a common trunk before it joined the deep femoral vein to form the common femoral vein; it was accompanied by a collateral canal which united with the superficial femoral vein before it joined the deep femur in 1 case (Figure 4, left side); and in two cases she received as a tributary a muscular vein of the quadriceps.

\subsection{Deep Femoral Vein}

The diameter of the deep femoral vein (DFV) was $7.60 \mathrm{~mm}$ (range: 2 and 13) The modal arrangement (circumflex femoral veins and quadriceps veins were tributaries of the deep femoral vein) was noted in 4 cases, i.e. $16.67 \%$. The anatomical variations noted were the absence of tributaries in the deep femoral vein in 20 cases $(83.33 \%)$.

\subsection{Common Femoral Vein}

Dimensions:

The common femoral vein had a mean diameter of $13.95 \mathrm{~mm}$ (range: 11 and $17 \mathrm{~mm}$ ). Its average length was $80.70 \mathrm{~mm}$ (extremes: 16 and $116 \mathrm{~mm}$ ).

Table 1. Distribution of anatomical subjects by sex.

\begin{tabular}{ccc}
\hline Sex & Effective & Pourcentage \\
\hline F & 3 & 25 \\
M & 9 & 75 \\
Total & 12 & 100 \\
\hline
\end{tabular}

In our study, we performed 24 dissections in 12 cadavers ( 9 men and 3 women).

Table 2. Distribution of anatomical subjects by size.

\begin{tabular}{ccc}
\hline Size (in millimeter) & Effective & Pourcentage \\
\hline $1.57-1.70$ & 4 & 33.33 \\
$1.71-1.82$ & 8 & 66.67 \\
Total & 12 & 100
\end{tabular}

The average size of the anatomical subjects was $1.73 \pm 7.01 \times 10^{-2} \mathrm{~mm}$ (extreme: 1.57 et 1.81 ). 
Mode of birth:

The modal disposition (the common femoral vein is formed by the union of the two superficial and deep femoral veins) was noted in 22 cases, i.e. $91.67 \%$. Anatomic variations were noted in 2 cases (8.33\%): the common femoral vein was formed by the union of 4 veins ( 2 veins of the quadriceps and the two superficial and deep femoral veins) in 1 case and in the other case it was formed by the meeting of 3 veins (a vein of the quadriceps and both femoral veins superficial and deep).

Affluent:

The common femoral vein received tributaries in the proportions indicated in Table 3.

The modal disposition (the great saphenous vein was the only tributary of the common femoral vein) was noted in 4 cases (16.67\%). The anatomical variations were noted in 20 cases (83.33\%): the most frequent of these variations were: the large saphenous vein (LSV), the lateral circumflex vein of the thigh (LCVT), the medial circumflex vein of the thigh (MCVT) and the quadriceps vein $(\mathrm{QV})$ flung themselves isolated in the common femoral vein in 7 cases (29.20\%) (Figure 1 on the right, Figure 3 on the left, Figure 4 to the left). Then, the variation which came in 2 nd position in term of frequency was: the common femoral vein received as tributaries, the LSV, the MCVT and a common core with LCVT and QV in 4 cases (16.67\%) (Figure 4 on the right); then in 3rd place came: LSV and a common core at LCVT, MCVT and QV were tributaries of the common femoral vein in 2 cases (8.33\%) (Figure 3 on the right); and LSV, 2 LCVT, MCVT and QV were tributaries of CFV in 2 cases (8.33\%); finally, the other variations were present in a proportion of 1 out of 24 cases, i.e. $4.16 \%$.

In our study, we observed that the MCVT, just after release between the pectin and psoas muscles, threw itself into the posterior aspect of the femoral vein

Table 3. Tributaries of the common femoral vein.

\begin{tabular}{ccc}
\hline Tributaries of common femoral vein & Effective & Pourcentage \\
\hline LSV & 4 & 16.67 \\
LSV, common core (LCVT, MCVT and QV) & 2 & 8.33 \\
LSV, MCVT et tronc commun aux (LCVT and QV) & 4 & 16.67 \\
LSV, LCVT, MCVT, and QV & 7 & 29.20 \\
LSV, LCVT, MCVT, QV et SICV & 1 & 4.16 \\
LSV, LCVT, 2 MCVT and QV & 1 & 4.16 \\
LSV, LCVT, MCVT, 2 QV and SICV & 1 & 4.16 \\
LSV, 2 LCVT, 2 MCVT & 1 & 4.16 \\
LSV, MCVT? tronc commun aux (QV et 2 LCVT) and QV & 1 & 4.16 \\
LSV, 2 LCVT, MCVT et 2 QV & 2 & 8.33 \\
Total & 24 & 100 \\
\hline
\end{tabular}

LCVT = lateral circumflex vein of the thigh, $\mathrm{LSV}=$ large saphenous vein, $\mathrm{MCVT}=$ medial circumflex vein of the thigh, $\mathrm{QV}=$ quadriceps vein, $\mathrm{SICV}=$ superficial iliac circumflex vein. 


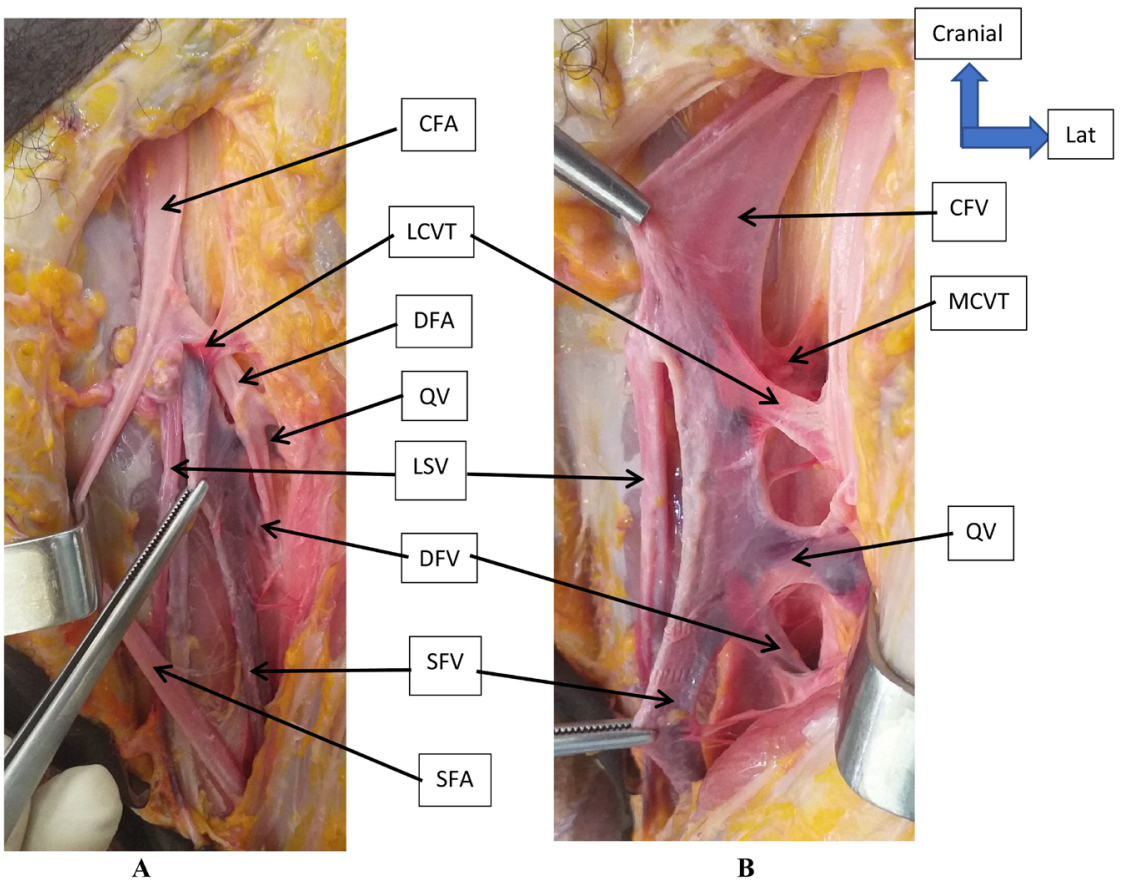

Figure 1. The right femoral veins. (A) (femoral veins with femoral arteries in place): LCVT went ahead of the DFA, QV goes back from the DFA; (B) (femoral vein after resection of femoral arteries): VFC is formed by the meeting of SFV and of DFV, isolated outbreak of QV, LCVT, MCVT and LSV in the CFV. CFA = common femoral artery, CFV = common femoral vein, DFA = deep femoral artery, DFV = deep femoral vein, LCVT = lateral circumflex vein of the thigh, LSV = large saphenous vein, MCVT = medial circumflex vein of the thigh, $\mathrm{QV}=$ quadriceps vein, $\mathrm{SFA}=$ superficial femoral vein, $\mathrm{SFV}=$ superficial femoral vein.

(Figure 1 on the right), it was split in 2 cases. The LCVT in its course in the femoral trunk passed below the bifurcation of the common femoral artery, between the superficial femoral artery in front and the deep femoral artery (DFA) in 23 cases (95.83\%) (Figure 2), in 1 case where 2 LCVT were thrown into the CFV in isolation, one passed in front of the DFA and the other passed back from the DFA, it was split in 15 cases (62.50\%) (Figure 2). The contribution of the quadriceps veins with the arteries was also described, the veins of the quadriceps passed in front of the DFA in 21 cases (87.50\%) (Figure 2); in 2 cases they passed behind the DFA (Figure 1 on the left) and in 1 case one of the veins of the quadriceps passed behind the DFA, the others passed in front of the DFA.

In all cases where the QV, LCVT and MCVT were isolated in the CFV, they were arranged in this order from bottom to top (Figure 1 on the right, Figure 3 on the left); except in a case where they were arranged in the following manner from bottom to top: the MCVT, the QV and the LCVT (Figure 3 on the left). The first provision was also present in cases where these veins formed a common trunk before reaching into the VFC (Figure 3 on the right, Figure 4 on the right).

\section{Discussion}

Very few studies have been performed on femoral veins especially by anatomic 


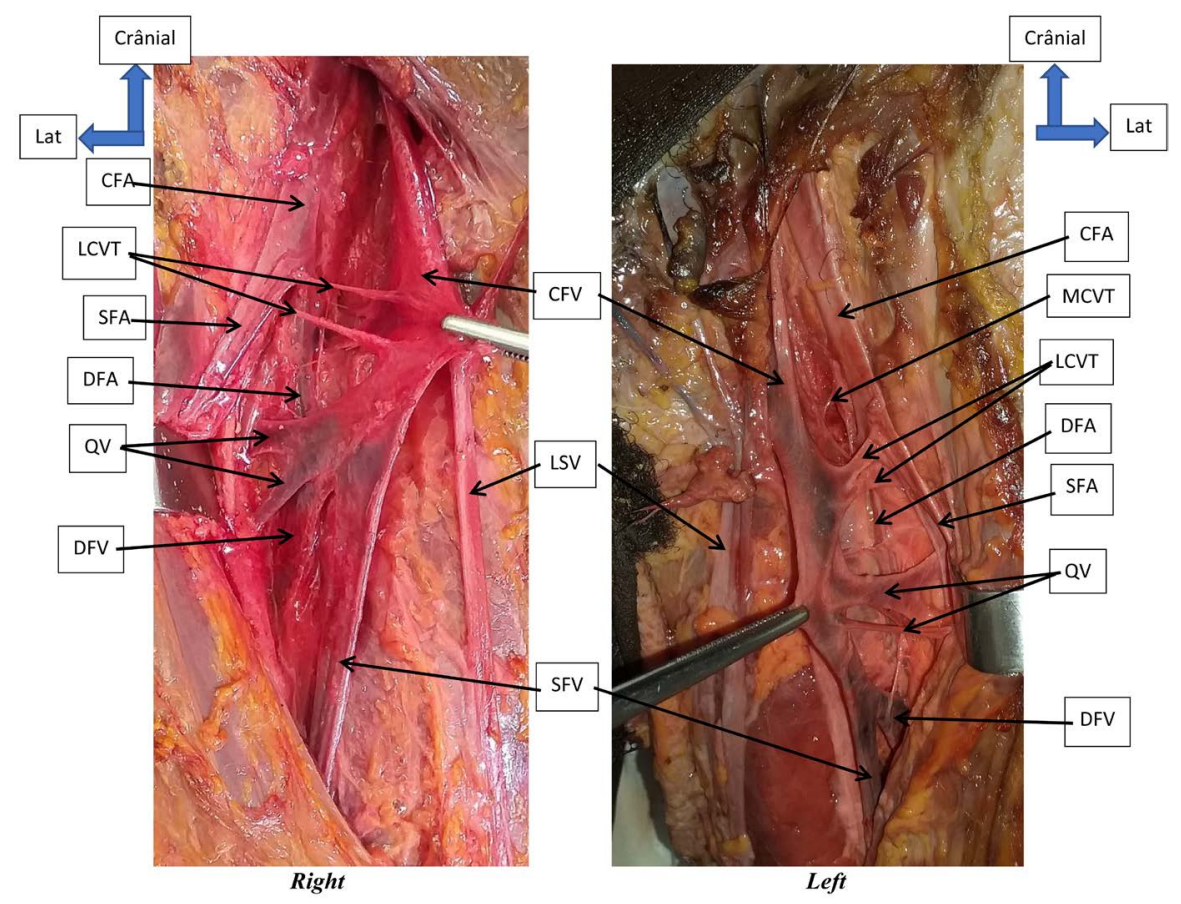

Figure 2. Femoral vein on both sides of a subject. Right: CFV is formed by the meeting of 4 vein (2 VQ, DFV and SFV). The QV and the LCVT went ahead of the DFA. Left: CFV is formed by the meeting of the DFV and the SFV, the QV and LCVT went ahead of the DFA. CFA = common femoral artery, CFV = common femoral vein, DFA = deep femoral artery, DFV = deep femoral vein, LCVT = lateral circumflex vein of the thigh, LSV = large saphenous vein, $\mathrm{MCVT}=$ medial circumflex vein of the thigh, $\mathrm{QV}=$ quadriceps vein, $\mathrm{SFA}=$ superficial femoral vein, $\mathrm{SFV}=$ superficial femoral vein.

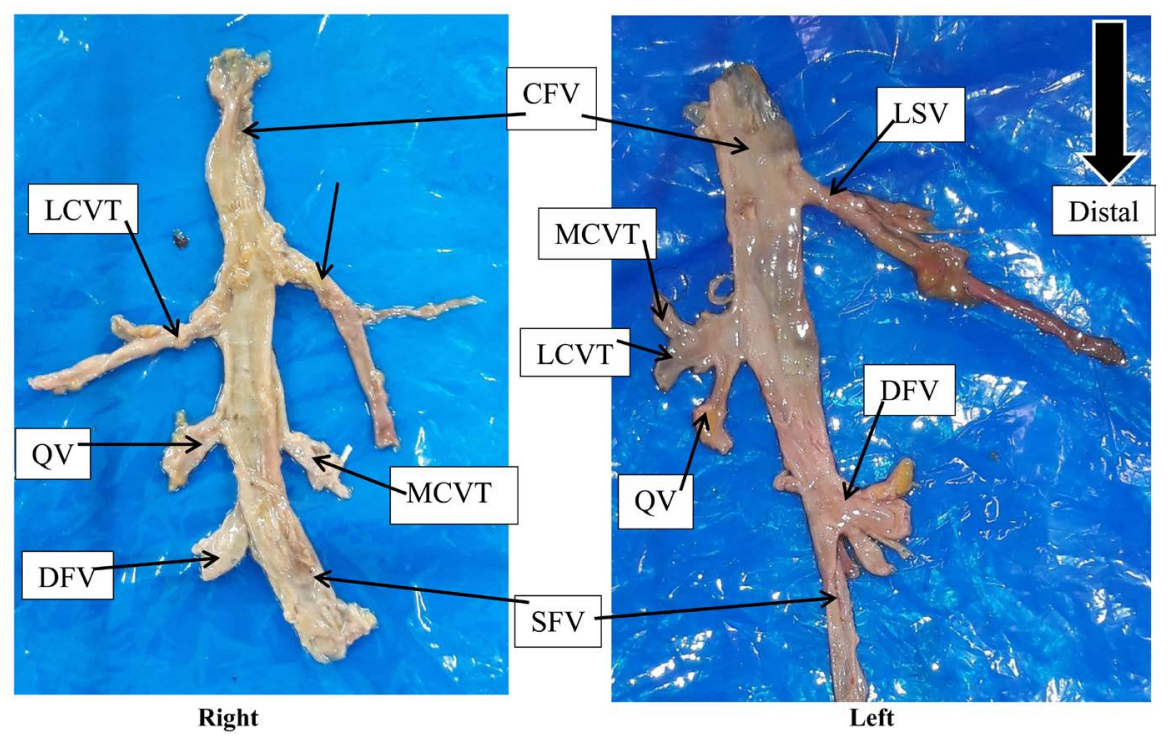

Figure 3. Femoral vein on both sides of a subject. Right: From the bottom up, threw themselves into the CFV: MCVT, QV, LCVT and LSV. Left: QV, LCVT and MCVT jumped into the CFV forming a common core. CFV = common femoral vein, DFV = deep femoral vein, LCVT = lateral circumflex vein of the thigh, LSV = large saphenous vein, $\mathrm{MCVT}=$ medial circumflex vein of the thigh, $\mathrm{QV}=$ quadriceps vein, $\mathrm{SFV}=$ superficial femoral vein. 


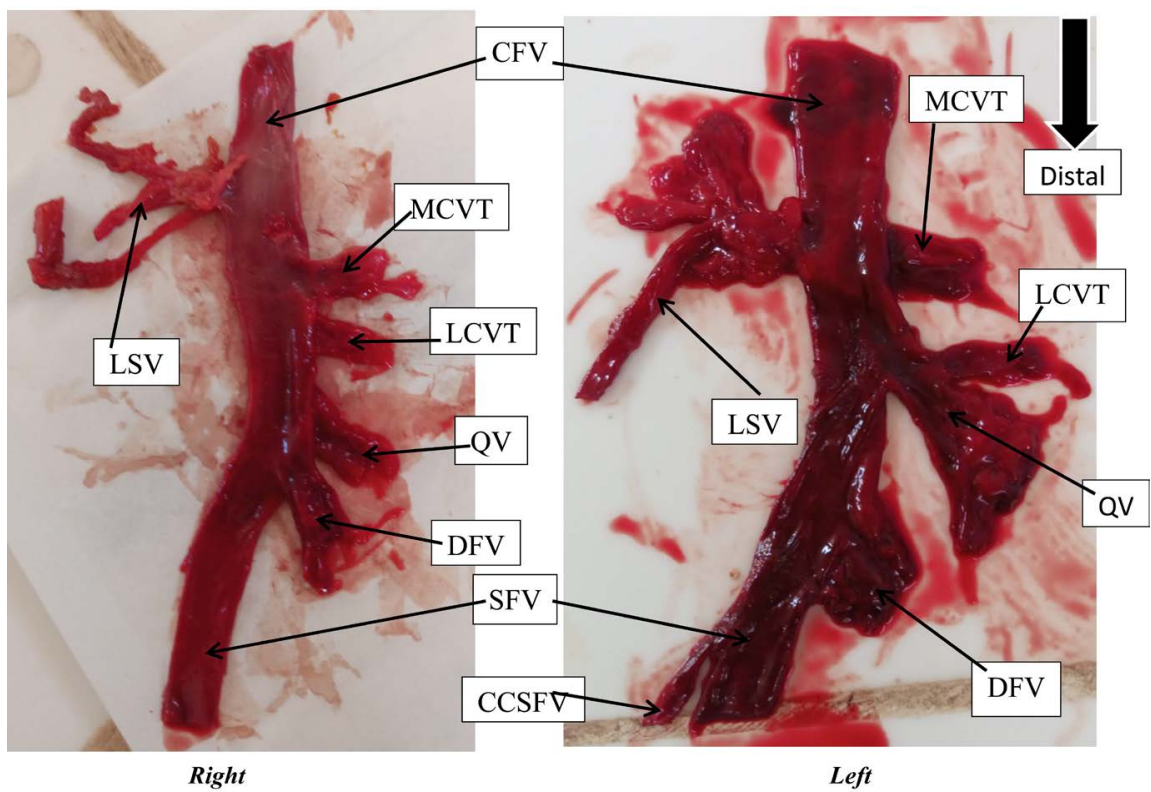

Figure 4. Femoral vein on both sides of a subject. Right: From bottom to top, were added in isolation in the CFV: QV, LCVT, MCVT and LSV. Left: QV and LCVT threw themselves into the CFV forming a common core. CCSFV = collateral canal of the superficial femoral vein, $\mathrm{CFV}=$ common femoral vein, $\mathrm{DFV}=$ deep femoral vein, $\mathrm{LCVT}=$ lateral circumflex vein of the thigh, LSV = large saphenous vein, MCVT = medial circumflex vein of the thigh, $\mathrm{QV}=$ quadriceps vein, $\mathrm{SFV}=$ superficial femoral vein.

dissection. Kamina [2] does not distinguish the common femoral vein from the superficial femoral vein. He describes these two veins as a single venous trunk called the femoral vein, which receives as affluent the deep femoral vein and the great saphenous vein. According to Bouchet and Cuilleret [1], there are three femoral veins: superficial, deep and common. In our study, we divided the femoral veins into three as did Bouchet and Cuilleret to facilitate the description of anatomical variations and comparison.

Dimensions: The dimensions of the femoral veins have not been described by many authors. According to Kamina [2], the femoral vein has a caliber of $9 \mathrm{~mm}$. In our study, the diameter of the superficial, deep and common femoral veins was $8.75 \mathrm{~mm} ; 7.60 \mathrm{~mm}$ and $13.95 \mathrm{~mm}$. The common femoral vein was $80.70 \mathrm{~mm}$ long.

Superficial femoral vein: Kamina [2] noted the splitting of the femoral vein. According to Cruveilhier [3], there are, for the half or two thirds of the femoral vein, one or two collateral channels, which run parallel to its direction. In our series, we noted the splitting of the superficial femoral vein in 2 cases, the SFV was accompanied in one case by a collateral canal (Figure 4 on the right) and it received as tributary a quadricipital vein in 2 cases.

Deep Femoral Vein: According to Kamina [2], DFV drains the medial and lateral circumflex veins of the thigh, femoral perforating veins and muscle twigs. According to Rouvière [4], the DFV before its termination receives numerous collateral branches, among which the quadricipital and anterior circumflex veins 
(lateral circumflexes). According to Bouchet and Cuilleret [1], the DFV receives close to its termination the circumflex veins and veins of the quadriceps. In our series, the DFV received in the femoral trigone, quadricipital veins, medial and lateral femoral circumflex veins of the thigh in 4 cases or $16.20 \%$. The remaining $83.80 \%$ were considered variations in which the DFV did not receive any affluent in the TF.

Common femoral vein: Length: According to Cruveilhier [3], the deep femoral vein opens into the femoral vein 2 to $3 \mathrm{~cm}$ below the inguinal ligament, which is the length of the common femoral vein. According to Moore and Dalley [4], the deep vein of the thigh emerges into the femoral vein at a distance of approximately $8 \mathrm{~cm}$ below the inguinal ligament. In our study, this length was $80.70 \mathrm{~mm}$ (extremes: 16 and $116 \mathrm{~mm}$ ), it was comparable to that of Moore and Dalley [4], on the other hand, it was larger than that found by Cruveilhier [3] 3 to 4 times.

Birth Mode: According to Bouchet and Cuilleret [1], the DFV meets at the SFV to form the CFV. In our study, CFV was formed by the union of superficial and deep femoral veins in 22 cases, in 91.17\% (Figure 1 and Figure 2 on the right). Anatomic variations were noted in 2 cases: in 1 case the CFV was formed by the union of 2 quadricipital veins, SFV and DFV (Figure 2 on the left); in the other case, it was formed by the union of a quadricipital vein, DFV and SFV.

Tributaries: According to Bouchet and Cuilleret [1], the CFV receives only one tributary which is the LSV. In our series, the LSV was the only tributary of the CFV in 4 cases or $18.18 \%$. The anatomical variations were noted in $81.82 \%$ in which the CFV received in addition to the LSV other veins (QV, LCVT, MCVT and superficial iliac circumflex vein) (Figures 1-4) in proportions indicated in Table 3. These variations have not been described in the literature. They are explained by the fact that the DFV unites early with the SFV, therefore, the longer length of the CFV.

According to Rouvière [5], the quadricipital veins and anterior circumflex veins (lateral circumflex veins of the thigh), to gain the venous trunk, most often cross the anterior surface of the deep femoral artery (DFA) and occupy the bifurcation angle formed by femoral and DFA. According to Bouchet and Cuilleret, the circumflex veins and veins of the quadriceps cross the anterior surface of the DFA. In our study, in all 23 cases (95.83\%), the LCVT passed ahead of the DFA (Figure 1 and Figure 2), in 1 case one of the LCVT passed behind the DFA; the quadricipital veins passed in front of the DFV in 21 cases (87.50\%) (Figure 2), in 2 cases (8.33\%) they passed behind the DFA (Figure 1 to left) and in 1 case $(4.16 \%)$ one of the veins of the quadriceps passed in front of the DFA, the others passed backwards.

\section{Conclusion}

The diameter of the femoral veins is important. The main tributaries of the common femoral vein are LSV, QV and circumflex femoral veins. Femoral vari- 
ations are numerous: $20.83 \%$ at SFV level, 83.33 at DFV level, $8.33 \%$ at birth level of CFV and $83.33 \%$ at tributaries of CFV.

\section{Acknowledgements}

We thank Professor Seydou Doumbia for his financial support to the article as well as the professors Nouhoun Ongoïba, Tièman Coulibaly and Sekou Sidibé for the knowledge they gave us and their encouragement.

\section{Conflicts of Interest}

The authors declare no conflicts of interest regarding the publication of this paper.

\section{References}

[1] Bouchet, A. and Cuilleret, J. (1996) La région inguino-fémorale. In: Anatomie topographique descriptive et fonctionnelle. Tome $3 \mathrm{~b}$ le membre inférieur. 3rd Edition, SIMEP Paris, 1487-1508.

[2] Kamina, P. (2009) Précis d'anatomie clinique anatomie générale, organogénèse des membres. Tome 1 membre supérieur membre inférieur. Maloine, Paris.

[3] Cruveilhier, J. (1871) Traité d'anatomie descriptive. Tome 3 Angéilogie, nevrologie. 4th Edition, Asselin P, Paris.

[4] Moore, K.L. and Dalley, A.F. (2007) Anatomie médicale: Aspects fondamentaux et applications cliniques (Dhem A \& Milaire J, Trad.). 5th Edition, De Boeck, Paris.

[5] Rouvière, H. and Delmas, A. (1991) Vaisseaux du membre inférieur. In: Anatomie humaine descriptive, topographique et fonctionnelle. Tome III Membres, système nerveux central. Treizième édition révisée et augmentée, Masson, Paris, 443-468. 